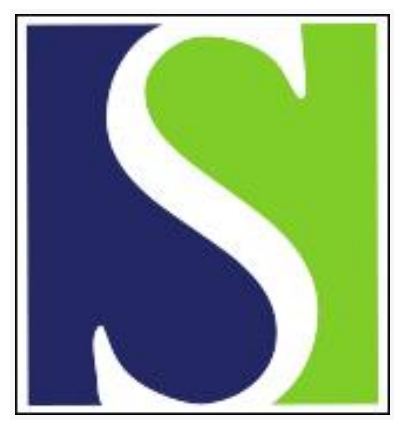

Scand J Work Environ Health 2017;43(5):393-395

https://doi.org/10.5271/sjweh.3657

Published online: 28 Jun 2017, Issue date: 01 Sep 2017

Analytical strategies to determine whether job strain is an important risk factor for occurrence of low-back pain by Burdorf $A$, LaMontagne $A D$

Affiliation: Department of Public Health, Erasmus MC, PO Box 2040. 3000 CA Rotterdam. The Netherlands. a.burdorf@erasmusmc.na.burdorf@erasmusmc.nl

Refers to the following texts of the Journal: 2015;41(2):107-110 2017;43(1):68-74 2016;42(6):481-489 2017;43(1):50-58 2017;43(5):396-404

The following articles refer to this text: 2018;44(4):394-402; 2019;45(3):215-216

Key terms: analytical strategy; back pain; cross-lagged panel analysis; editorial; fixed effect; job strain; low-back pain; pain; risk factor; structural equation modeling; temporal relationship

This article in PubMed: www.ncbi.nlm.nih.gov/pubmed/28657639 


\section{Analytical strategies to determine whether job strain is an important risk factor for occurrence of low-back pain}

Low-back pain (LBP) is one of the most prevalent health problems in occupational populations. Although still fiercely debated (1), several biomechanical factors have been consistently acknowledged as important risk factors for LBP, most notably heavy lifting, awkward back postures due to severe bending and rotating, and whole body vibration (2-4). In the quest to unravel the multidimensional origin of work-induced LBP, the jury is still out on the particular contribution of psychosocial factors at work. Some authors have reported that high psychosocial work demands is an independent risk factor of LBP (2), whereas others have noted that psychosocial factors, such as job strain, are more important in aggravation and slower recovery of LBP (5). Moreover, authors have also focused on job strain as a promoting factor of sickness absence among those with musculoskeletal complaints (6).

The uncertainty as to the particular contribution of psychosocial factors to LBP undoubtedly partly stems from differences between the conceptual models used to unravel the temporal and causal relationships between these factors and LBP. In this issue of the Scandinavian Journal of Work, Environment \& Health, Magnusson Hanson and colleagues address this topic with a novel approach by combining structural equation modelling (SEM) with a fixed-effects (FE) regression in a longitudinal study with four waves over a 6-year period (7).

Although SEM models have not been very popular in epidemiology, in part due to the required assumptions (8), their ability to construct a quantitative causal diagram has a certain appeal. The most complex SEM model in the current article estimates the bidirectional associations between job strain and subsequent LBP in successive waves and between LBP and subsequent job strain (cross-lagged paths), while controlling for autocorrelation and adjusting for important confounders. In this approach, temporal and causal associations are investigated, and the presence of reversed causality can be refuted. The SEM model shows that job strain predicts occurrence and severity of LBP two years later. So far, so good, the reader will think. Isn't this what we would have expected? Interestingly, the analysis also shows that an increase in LBP severity predicts later job strain! This may leave the reader dumbfounded. What is the cause and what is the consequence? Is this reversed association caused by unmeasured individual characteristics? Is this association a clear indication of common source bias since both variables are self-reports?

The innovation in this article is that the authors have incorporated a FE regression into the SEM models. FE models provide an excellent solution for removing time-invariant confounding by using each person as his or her own control and focus solely on within-person change $(9,10)$. The essence of a FE model is that it relates change in exposure to change in health outcome within individuals, thereby controlling for all measured and unmeasured individual characteristics and other potential confounders (eg, aspects of work or home environment) that do not vary over time. This will remove, for example, common source (dependent misclassification) bias. The attractiveness of the FE approach has been illustrated in recent studies on mental health at work $(11,12)$.

The SEM model and the SEM-FE model show different results. Whereas in the SEM model, bidirectional associations between job strain and LBP are identified, in the SEM-FE model these associations are no longer present. This intriguing difference in findings between analytical techniques requires careful interpretation. As the authors propose themselves, a first explanation may be that the SEM-FE model 
suggests that associations between job strain and LBP may be due to residual confounding by time invariant characteristics, such as the individual's tendency to respond similarly to different questions in a questionnaire. An alternative explanation is that both models essentially present different information on the exposure-response relation between job strain and LBP. The FE component in the SEM-FE model only includes within-person effects whereby the effect of job strain on LBP is based only on those participants who experienced a change in exposure during the period of observation. In essence, this estimates the short-term effects of a change in job strain on the change in occurrence of LBP. The traditional SEM model, however, includes both within- and between-person effects and it may be hypothesized that cumulative exposure to high job strain over all waves during the study may increase the likelihood of having LBP. A third explanation relates to the study population in both analytical strategies. Whereas the traditional SEM model includes all workers who responded to the four consecutive questionnaires, the FE component only uses participants who experienced one or more changes in exposure status. As the authors reported, approximately $61 \%$ of all workers had a stable exposure to job strain and, hence, these workers do not contribute to the estimated within-person changes. This is certainly a limitation of a FE model; it will reduce power and generalizability compared to standard SEM models.

The rapidly increased use of FE models in epidemiology is spurred in part by the debate on causal inference. The FE approach is valuable for optimizing causal inference but does not tell the whole story of an exposure-outcome relationship. It is well established, for example, that sustained exposure to high job strain is associated with a number of adverse health outcomes, such as mental health and cardiovascular disease, yet such sustained adverse exposure is not represented in a FE coefficient. The two main candidates for explaining differences between a FE model and statistical methods that include both within- and between-person variation (ordinary least squares, random effects regression) are bias and associations of short-term versus sustained exposure. Each of these approaches has their strengths and can be used in combination to answer complementary research questions (11).

Magnusson Hanson and colleagues must be applauded for their innovative approach to analyse the associations between job strain and LBP. They demonstrate eloquently that the analytical choice has a profound impact on the results. This raises important questions on the validity of many observational studies on the influence of psychosocial factors on LBP. In this particular situation, the mundane statement that further research is needed is highly appropriate. The application of FE models in occupational epidemiology will offer new insights into temporal and causal associations between exposure and health effects.

\section{References}

1. Van Dieën JH, Kuijer PPFM, Burdorf A, Marras WS, Adams MA. Non-specific low-back pain, Lancet 2012;379:1874. https://doi.org/10.1016/S0140-6736(12)60803-4

2. Andersen LL, Fallentin N, Ajslev JZ, Jakobsen MD, Sundstrup E. Association between occupational lifting and dayto-day change in low-back pain intensity based on company records and text messages. Scand J Work Environ Health 2017;43(1):68-74. https://doi.org/10.5271/sjweh.3592

3. Da Costa BR, Vieira ER. Risk factors for work-related musculoskeletal disorders: A systematic review of recent longitudinal studies. Am J Ind Med. 2010;53(3):285-323.

4. Griffith LE, Shannon HS, Wells RP, Walter SD, Cole DC, Côté P, et al. Individual participant data meta-analysis of mechanical workplace risk factors and low-back pain. Am J Public Health. 2012;102(2):309-18. https://doi.org/10.2105/ AJPH.2011.300343

5. Petit A, Fouquet N, Roquelaure Y. Chronic low-back pain, chronic disability at work, chronic management issues. Scand J Work Environ Health 2015;41:107-10. https://doi.org/10.5271/sjweh.3477

6. Sommer TG, Svendsen SW, Frost P. Sickness absence and permanent work disability in relation to upper- and lowerbody pain and occupational mechanical and psychosocial exposures. Scand J Work Environ Health. 2016;42(6):481-9. https://doi.org/10.5271/sjweh.3600 
7. Magnusson Hanson LL, Madsen IEH, Rugulies R, Peristera P, Westerlund H, Descatha A. Temporal relationships between job strain and low-back pain. Scand J Work Environ Health. 2017;43(5):396-404. https://doi.org/0.5271/sjweh.3654

8. VanderWeele T. Structural equation models and epidemiologic analysis. Am J Epidemiol 2012;176(7):608-12. https://doi. org/10.1093/aje/kws213

9. Gunasekara FI, Richardson K, Carter K, Blakely T. Fixed effects analysis of repeated measures data. Int J Epidemiol 2014;43(1):264-9. https://doi.org/10.1093/ije/dyt221

10. Hirai AS, Kaufman JS. Fixed versus random effects models for multilevel and longitudinal data (Chapter 15). In: Oakes JM, Kaufman JS, editors. Methods in social epidemiology, 2nd Edition. San Francisco: Jossey-Bass, 2017.

11. Milner A, LaMontagne AD. Underemployment and mental health: comparing fixed-effects and random-effects regression approaches in an Australian working population cohort. Occup Environ Med 2017;74(5):344-50. https://doi.org/10.1136/ oemed-2016-103706

12. Milner A, Krnjacki L, LaMontagne AD. Psychosocial job quality and mental health among young workers: a fixed-effects regression analysis using 13 waves of annual data. Scand J Work Environ Health 2017;43(1):50-8. https://doi.org/10.5271/ sjweh.3608

Alex Burdorf

Department of Public Health, Erasmus MC, PO Box 2040, 3000 CA Rotterdam, The Netherlands [email: a.burdorf@erasmusmc.nl]

\author{
Anthony D LaMontagne \\ Centre for Population Health Research, \\ Deakin University, \\ 221 Burwood Highway, Burwood, VIC 3125, \\ Australia \\ [email: tony.lamontagne@deakin.edu.au]
}

